\title{
HIERARCHIES OF FUNCTION CLASSES DEFINED BY THE FIRST-VALUE OPERATOR
}

\begin{abstract}
Armin HemmerLing ${ }^{1}$
Abstract. The first-value operator assigns to any sequence of partial functions of the same type a new such function. Its domain is the union of the domains of the sequence functions, and its value at any point is just the value of the first function in the sequence which is defined at that point. In this paper, the first-value operator is applied to establish hierarchies of classes of functions under various settings. For effective sequences of computable discrete functions, we obtain a hierarchy connected with Ershov's one within $\Delta_{2}^{0}$. The non-effective version over real functions is connected with the degrees of discontinuity and yields a hierarchy related to Hausdorff's difference hierarchy in the Borel class $\Delta_{2}^{\mathrm{B}}$. Finally, the effective version over approximately computable real functions forms a hierarchy which provides a useful tool in computable analysis.
\end{abstract}

Mathematics Subject Classification. 03D55, 03D65, 03E15, $03 \mathrm{~F} 60$.

\section{InTRODUCTION AND BASIC NOTIONS}

Let be given a finite or transfinite sequence $\mathcal{F}=\left(f_{\xi}\right)_{\xi<\alpha}$ of partial functions of the same type. This means that $f_{\xi}: A \succ B$, for some source set $A$ and a target $B, \alpha$ is an ordinal number, and $\xi$ runs through the set $\{\xi: \xi<\alpha\}=\{\xi: \xi \in$ $\alpha\}=\alpha$. Partial functions, $f: A \succ B$, are here understood as special subsets of Cartesian products, $f \subseteq A \times B$. By $f(x) \downarrow$ we denote that $f(x)$ exists, i.e., $x \in \operatorname{dom}(f)$, whereas $f(x) \uparrow$ or $f(x) \simeq \uparrow$ means that $x \notin \operatorname{dom}(f)$.

Keywords and phrases. Hierarchies of functions, degree of discontinuity, computable analysis, effective descriptive set theory, Hausdorff hierarchy, Ershov hierarchy.

1 Ernst-Moritz-Arndt-Universität Greifswald, Institut für Mathematik und Informatik,

Friedrich-Ludwig-Jahn-Str. 15a, 17487 Greifswald, Germany; hemmerli@uni-greifswald.de 
The first-value operator, $\Phi$, assigns to a sequence $\mathcal{F}$ the function $\Phi(\mathcal{F})=\bar{f}$, $\bar{f}: A \longleftrightarrow B$, defined by

$\bar{f}(x) \simeq\left\{\begin{array}{cl}f_{\xi_{x}}(x) & \text { if there is a } \xi<\alpha \text { with } f_{\xi}(x) \downarrow, \text { and } \xi_{x}=\min \left\{\xi<\alpha: f_{\xi}(x) \downarrow\right\}, \\ \uparrow & \text { if } f_{\xi}(x) \uparrow \text { for all } \xi<\alpha .\end{array}\right.$

This technique was introduced by Epstein et al. [3] in order to describe the Ershov hierarchy of classes of discrete sets $M \subseteq \mathbb{N}^{k}$, even if the operator $\Phi$ was not explicitly defined there. In [8,9], we transferred this idea to Hausdorff's hierarchy establishing a substructure of the Borel class $\Delta_{2}^{\mathrm{B}}$, as well as to the HausdorffErshov hierarchy within $\Delta_{2}^{\text {ta }}$, the effective counterpart of $\Delta_{2}^{\mathrm{B}}$. In all these cases, emphasis was put on functions of type $f: A \longleftrightarrow\{0,1\}$ characterizing sets $M_{f} \subseteq$ $A$ by $M_{f}=\{x: f(x)=1\}$. The level of a set $M$ in the related hierarchy is determined by the (minimal) length $\alpha$ of a sequence $\mathcal{F}$ consisting of functions of some basic type and characterizing the set $M$ as $M=M_{\Phi(\mathcal{F})}$. To ensure features of effectivity, the sequences $\mathcal{F}$ under consideration have to fulfill related requirements of computability. In particular, the order types $\alpha$ have to be constructive ordinals then.

In the present paper, our interest is directed to classes of functions themselves. The (minimal) length of sequences, which is needed to obtain a function by means of the first-value operator from some basic class $\mathfrak{F}$, determines the level (or degree) of that function w.r.t. $\mathfrak{F}$. It will turn out that this approach both yields a unifying view to some well-known concepts of classical computability theory and descriptive set theory, as well as it leads to some new features and tools concerning subjects of effective analysis.

We begin with explaining the non-effective basic version of the first-value hierarchies. Let $\mathfrak{F}$ be a non-empty set of partial functions $f: A \longleftrightarrow B$, for fixed sets $A$ and $B$. Then, for any ordinal number $\alpha$, we put

$\nabla_{\alpha}(\mathfrak{F})=\left\{\Phi(\mathcal{F}): \mathcal{F}=\left(f_{\xi}\right)_{\xi<\alpha}\right.$ is a sequence of functions, where $f_{\xi} \in \mathfrak{F}$ for all $\left.\xi<\alpha\right\}$

So we always have $\nabla_{0}(\mathfrak{F})=\{\emptyset\}$, the singleton consisting of the empty function $\emptyset$, and $\nabla_{1}(\mathfrak{F})=\mathfrak{F}$. Moreover, it is easily shown that

$$
\nabla_{\alpha+1}(\mathfrak{F})=\left\{f: f(x) \simeq\left\{\begin{array}{ll}
g(x) & \text { if } g(x) \downarrow, \\
h(x) & \text { otherwise, }
\end{array} \quad \text { for functions } g \in \nabla_{\alpha}(\mathfrak{F}), h \in \mathfrak{F}\right\}\right.
$$

If $\emptyset \in \mathfrak{F}$, then $\nabla_{\alpha}(\mathfrak{F}) \subseteq \nabla_{\beta}(\mathfrak{F})$ whenever $\alpha \leq \beta$. Indeed, given an $\alpha$-sequence $\mathcal{F}=\left(f_{\xi}\right)_{\xi<\alpha}$, we obtain a $\beta$-sequence $\mathcal{F}^{\prime}=\left(f_{\xi}^{\prime}\right)_{\xi<\beta}$ with $\Phi(\mathcal{F})=\Phi\left(\mathcal{F}^{\prime}\right)$ by putting $f_{\xi}^{\prime}=f_{\xi}$, for $\xi<\alpha$, and $f_{\xi}^{\prime}=\emptyset$, for $\alpha \leq \xi<\beta$. If $\mathfrak{F}$ contains a total function $\tilde{f}$, then any partial function $f \in \nabla_{\alpha}(\mathfrak{F})$ is a restriction of a total function from $\nabla_{\alpha+1}(\mathfrak{F})$. To show this, a sequence defining $f$ is simply enlarged by $\tilde{f}$.

If $\bigcup_{\eta<\xi} \operatorname{dom}\left(f_{\eta}\right) \supseteq \operatorname{dom}\left(f_{\xi}\right)$, then the function $f_{\xi}$ does not influence the result of the sequence, $\Phi(\mathcal{F})$. Thus, given a sequence $\mathcal{F}=\left(f_{\xi}\right)_{\xi<\alpha}$, by transfinite recursion 
one can define a sequence $\mathcal{F}^{\prime}=\left(f_{\xi}^{\prime}\right)_{\xi<\alpha^{\prime}}$ such that $\alpha^{\prime} \leq \alpha, \bigcup_{\eta<\xi} \operatorname{dom}\left(f_{\eta}^{\prime}\right) \subset$ $\bigcup_{\eta \leq \xi} \operatorname{dom}\left(f_{\eta}^{\prime}\right)$ and $\Phi(\mathcal{F})=\Phi\left(\mathcal{F}^{\prime}\right)$. More precisely, $\mathcal{F}^{\prime}=\left(f_{\xi_{\eta}}\right)_{\eta<\alpha^{\prime}}$ with a suitably defined strictly increasing sequence $\left(\xi_{\eta}\right)_{\eta<\alpha^{\prime}}$ yielding $\mathcal{F}^{\prime}$ as a subsequence of $\mathcal{F}$. So, for countable universes $A$, we can restrict ourselves to sequences $\mathcal{F}$ whose lengths $\alpha$ belong to the second number class, $\mathrm{C}_{\mathrm{II}}$, which consists of all ordinal numbers of finite or countably infinite cardinality.

Also, if $A$ is a separable topological space and the basic functions $f \in \mathfrak{F}$ are supposed to have open domains, sequences of lengths $\alpha \in \mathrm{C}_{\mathrm{II}}$ are sufficient to obtain all possible results of the first-value operator applied to arbitrary sequences built of functions from $\mathfrak{F}$. Indeed, by $\left(\bigcup_{\eta<\xi} \operatorname{dom}\left(f_{\eta}\right)\right)_{\xi<\alpha}$ we obtain an increasing sequence of open subsets of $A$, and if it is supposed to be strictly increasing, its length $\alpha$ must belong to $\mathrm{C}_{\mathrm{II}}$. So, in all cases we will be interested in, the restriction to sequences of order types from $\mathrm{C}_{\mathrm{II}}$ is justified. In particular, all constructive ordinals belong to $\mathrm{C}_{\mathrm{II}}$ too.

The main subject of this paper is to explore properties of linear hierarchies $\left(\nabla_{\alpha}^{\cdots}(\mathfrak{F})\right)_{\alpha \in \mathrm{C}_{I I}}$, under several settings concerning the universes $A$ and the basic class $\mathfrak{F}$, possibly combined with requirements concerning the sequences $\mathcal{F}$ to which the operator $\Phi$ has to be applied. They will be indicated by upper labels at the $\nabla$-sign.

To give a first illustration, we consider the special case that $A=B=\mathbb{N}$, the set of natural numbers. Notice that $\mathbb{N}=\omega$. Nevertheless, we shall use both these denotations in the sequel: $\mathbb{N}$ if the set is mainly considered as a universe, $\omega$ if the viewpoint of ordinal number dominates. As basic classes of functions, $\mathfrak{F}$, we first consider

$$
\begin{aligned}
\mathfrak{F}_{\text {sing }} & =\{f: f: \mathbb{N} \succ \mathbb{N}, \operatorname{card}(f) \leq 1\} \quad \text { and } \\
\mathfrak{F}_{\text {fin }} & =\{f: f: \mathbb{N} \succ \mathbb{N}, \operatorname{card}(f)<\omega\} .
\end{aligned}
$$

Obviously, $\nabla_{n}\left(\mathfrak{F}_{\text {sing }}\right)=\{f: \operatorname{card}(f) \leq n\}$ and $\nabla_{n}\left(\mathfrak{F}_{\text {fin }}\right)=\mathfrak{F}_{\text {fin }}$ if $1 \leq n<\omega$, but $\nabla_{\alpha}\left(\mathfrak{F}_{\text {sing }}\right)=\nabla_{\alpha}\left(\mathfrak{F}_{\text {fin }}\right)=\{f: f: \mathbb{N} \succ \mathbb{N}\}$ for all $\alpha \geq \omega$.

Quite analogous results hold for functions of type $f: \mathbb{N}^{k} \succ \mathbb{N}, k \geq 1$, as well as for many other similar settings. So, the non-effective versions of first-value hierarchies over discrete universes collapse already at level $\omega$ with the largest possible class of partial functions, even for rather simple basic classes $\mathfrak{F}$.

To obtain more interesting results over discrete universes, the operator $\Phi$ will be restricted to effective sequences. This is the subject of the next section. Then we shall consider real-valued functions over Euclidean spaces, $f: \mathbb{R}^{k} \succ \mathbb{R}$. The non-effective setting studied in Section 3 provides the basic background of the effective version which will be elaborated in Section 4. Throughout this paper, we try to emphasize analogies and relationships between the three settings: effective discrete functions and non-effective resp. effective real functions.

This unifying view is just the main feature and motivation of the present paper. Most of the results and techniques are immediate adaptations from the related hierarchies of classes of pointsets, and some are even already known for function classes. Nevertheless, the treatment of these three settings in analogy to each 
other based on the first-value operator has not yet been done so far, but it surely contributes to a deeper understanding of the related hierarchies.

In the continuous settings, we restrict ourselves to the treatment of (real functions over) Euclidean spaces $\mathbb{R}^{k}$ with their natural topology. From the viewpoint of computable analysis, this seems to be the most interesting case. Hierarchies of classes of pointsets over the Cantor space and the Baire space were considered by Selivanov [20]. He already remarked "a deep and useful connection of the effective Hierarchy with limiting computations of a special kind". The latter means an implicit application of the first-value operator.

In the remaining part of this introductory section, we show how the $\mu$ operator of classical recursion theory can be substituted by the first-value operator $\Phi$. This gives a further illustration and demonstrates the computational power of the $\Phi$ operator. The reader who is preferably interested in the function hierarchies we announced may skip this part.

The operator $\mu$ assigns to any function $g: \mathbb{N}^{k+1} \succ \mathbb{N}, k \geq 1$, the $k$-ary function $\mu g$ defined by

$\mu g(\vec{x}) \simeq\left\{\begin{array}{cl}\min \{y: g(\vec{x}, y)=0\} & \text { if there is a } y \text { with } g(\vec{x}, y)=0 \\ \uparrow & \text { and } g\left(\vec{x}, y^{\prime}\right) \downarrow \text { for all } y^{\prime}<y\end{array}\right.$

By a partial primitive recursive (briefly: p.pr.r.) function $f: \mathbb{N}^{k} \succ \mathbb{N}$, we understand a restriction of a (total) primitive recursive function to a primitive recursive domain. This means that there are primitive recursive functions $g, h: \mathbb{N}^{k} \longrightarrow \mathbb{N}$ such that

$$
f(\vec{x}) \simeq\left\{\begin{array}{cl}
g(\vec{x}) & \text { if } h(\vec{x})=0 \\
\uparrow & \text { otherwise }
\end{array}\right.
$$

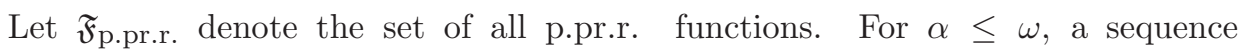

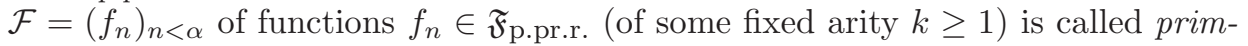
itive recursive (pr.r.) if there are primitive recursive functions $g, h: \mathbb{N}^{k+1} \longrightarrow \mathbb{N}$ such that

$$
f_{n}(\vec{x}) \simeq\left\{\begin{array}{cl}
g(\vec{x}, n) & \text { if } h(\vec{x}, n)=0 \\
\uparrow & \text { otherwise. }
\end{array}\right.
$$

Correspondingly, $\nabla_{\alpha}^{\text {pr.r. }}\left(\mathfrak{F}_{\text {p.pr.r. }}\right)$ is the class of all functions obtained by the firstvalue operator applied to primitive recursive sequences of length $\alpha$ of functions from $\mathfrak{F}_{\text {p.pr.r. }}$ Finally, let the class of all partial recursive functions be denoted by $\mathfrak{F}_{\text {p.r. }}$.

Lemma 1.1. $\nabla_{n}^{\text {pr.r. }}\left(\mathfrak{F}_{\text {p.pr.r. }}\right)=\mathfrak{F}_{\text {p.pr.r. }}$ if $1 \leq n<\omega$, and $\nabla_{\omega}^{\text {pr.r. }}\left(\mathfrak{F}_{\text {p.pr.r. }}\right)=\mathfrak{F}_{\text {p.r. }}$.

The first statement follows, since for finite sequences $\mathcal{F}, \Phi(\mathcal{F})$ can be expressed by a conditional equation with finitely many branches characterized by conditions which are conjunctions of finitely many primitive recursive predicates, so they are primitive recursive too. Inclusion " $\subseteq$ " of the second statement is also obvious 
from computability theory. To prove "卫", we use a weak version of Kleene's normal-form theorem. Given a partial recursive function $f: \mathbb{N}^{k} \succ \mathbb{N}$, there are (total) primitive recursive functions $g: \mathbb{N} \longrightarrow \mathbb{N}$ and $h: \mathbb{N}^{k+1} \longrightarrow \mathbb{N}$ such that $f=g \circ \mu h$. Let

$$
f_{n}(\vec{x}) \simeq\left\{\begin{array}{cl}
g(n) & \text { if } h(\vec{x}, n)=0 \\
\uparrow & \text { otherwise }
\end{array}\right.
$$

$\mathcal{F}=\left(f_{n}\right)_{n<\omega}$ is a primitive recursive sequence of p.pr.r. functions, and we have $f=\Phi(\mathcal{F})$.

So, in order to define the class of partial recursive functions from that of primitive recursive functions, instead of the $\mu$ operator, the first-value operator applied to primitive recursive sequences (each of which is determined by a p.pr.r. function) would be sufficient.

\section{THE ERSHOV HIERARCHY FOR DISCRETE FUNCTIONS}

Now we are going to study the first-value operator on effective sequences of computable functions of type $f: \mathbb{N}^{k} \succ \mathbb{N}$. Since $\mathbb{N}^{k}$ and $\mathbb{N}$ are recursively isomorphic via Cantor's $k$-tuple function, without loss of generality we could restrict ourselves to the case $k=1$. However, to remain in analogy to the later treatment of $k$-ary real functions, also here we prefer to consider arbitrary dimensions $k \in \mathbb{N}_{+}$.

As mentioned in Section 1, the first-value operator was essentially introduced in [3], within the recursion theoretic setting around Ershov's hierarchy. So the related notions and results are already well-known.

Effectivity for transfinite sequences is defined by means of constructive ordinals. We report some fundamental facts and denotations employed in the sequel. For more details, the reader may consult [19]. Let $\left(\phi_{n}: n \in \mathbb{N}\right)$ be a standard (Kleene) numbering of the partial recursive functions.

A naming system (of ordinals), $S$, is given by a numbering $\nu_{S}: \mathbb{N} \succ \mathrm{C}_{\mathrm{II}}$ such that

- $\operatorname{ran}\left(\nu_{S}\right)$ is an initial segment of ordinals: $\operatorname{ran}\left(\nu_{S}\right)=\alpha=\{\xi: \xi<\alpha\}$ for some $\alpha \in \mathrm{C}_{\mathrm{II}}$;

- for any $n \in \operatorname{dom}\left(\nu_{S}\right)$, it is decidable (by a partial recursive function $\left.k_{S}: \mathbb{N} \succ\{0,1,2\}\right)$ whether $\nu_{S}(n)=0$ or whether $\nu_{S}(n)$ is a successor or a limit number;

- for any number $n$, where $\nu_{S}(n)$ is a successor, a number $n^{\prime}$ is computable (by a partial recursive function $p_{S}$ ) such that $n \in \nu_{S}^{-1}\left(\nu_{S}\left(n^{\prime}\right)+1\right)$;

- and for any name $n \in \nu_{S}^{-1}(\lambda)$ of a limit number $\lambda$, an index $n^{\prime}$ is computable (by a partial recursive function $q_{S}$ ) such that $\phi_{n^{\prime}}$ is a total function and $\left(\nu_{S}\left(\phi_{n^{\prime}}(m)\right)_{m \in \mathbb{N}}\right.$ is an increasing sequence of ordinals converging to $\lambda$.

More precisely, we have $S=\left(\nu_{S}, k_{S}, p_{S}, q_{S}\right)$. An ordinal $\alpha \in \mathrm{C}_{\mathrm{II}}$ is called constructive iff there is a naming system $S$ for which $\alpha \in \operatorname{ran}\left(\nu_{S}\right)$. $S$ is called recursively related iff the set $\left\{\left(n, n^{\prime}\right): n, n^{\prime} \in \operatorname{dom}\left(\nu_{S}\right), \nu_{S}(n) \leq \nu_{S}\left(n^{\prime}\right)\right\}$ is recursive, it is 
called univalent iff $\nu_{S}$ is injective. To any constructive ordinal $\alpha$, there is a recursively related, univalent (briefly, r.r.u.) system $S$ assigning a name to that ordinal. So we can restrict ourselves to such special naming systems. They will be denoted in the form $\alpha / S$. Notice that each concept based on a constructive ordinal $\alpha$ might depend both on that ordinal and its r.r.u. naming system $S$ used in the corresponding context. Any univalent naming system $S$ with $\operatorname{ran}\left(\nu_{S}\right)=M \subseteq \mathbb{N}$ is computationally equivalent to the identical numbering $\nu=\operatorname{id}_{M}$. Thus, for $\alpha \leq \omega$ the canonical naming system with the identical mapping as numbering is always used, without loss of generality.

Given a naming system $S, S$-computability of functions like $f: \mathbb{N}^{k} \times \mathrm{C}_{\mathrm{II}} \succ \mathbb{N}$, $k \in \mathbb{N}$, is understood as usual w.r.t. the numbering $\nu_{S}$ : there is a recursive function $\varphi: \mathbb{N}^{k} \times \mathbb{N} \succ \mathbb{N}$ such that $\varphi(\vec{x}, n) \simeq f\left(\vec{x}, \nu_{S}(n)\right)$ for all $\vec{x} \in \mathbb{N}^{k}$ and $n \in \operatorname{dom}\left(\nu_{S}\right)$. For a naming system $\alpha / S$, an $\alpha$-sequence $\mathcal{F}=\left(f_{\xi}\right)_{\xi<\alpha}$ of functions $f_{\xi}: \mathbb{N}^{k} \succ \mathbb{N}$ is said to be $S$-computable iff there is an $S$-computable function $\bar{f}: \mathbb{N}^{k} \times \mathrm{C}_{\mathrm{II}} \longleftrightarrow \mathbb{N}$ such that $\bar{f}(\vec{x}, \xi) \simeq f_{\xi}(\vec{x})$ for all $\vec{x} \in \mathbb{N}^{k}$ and all ordinals $\xi<\alpha$. Then, if $S$ is recursively related, the domain of the function $\Phi(\mathcal{F})$ is r.e.: $\operatorname{dom}(\Phi(\mathcal{F}))=\bigcup_{\xi<\alpha} \operatorname{dom}\left(f_{\xi}\right) \in \Sigma_{1}^{0}$.

A function $f: \mathbb{N}^{k} \longleftrightarrow \mathbb{N}$ is called $\alpha / S$-recursive iff $f=\Phi(\mathcal{F})$ for an $S$ computable $\alpha$-sequence $\mathcal{F}$ of k-ary p.r. functions, and $f$ is said to be $\alpha$-recursive iff it is $\alpha / S$-recursive for some r.r.u. naming system $\alpha / S$. So we get the following classes of functions, in the notation-dependent and the notation-independent version, i.e., with and without "/S", respectively:

$$
\nabla_{\alpha(/ S)}^{\mathrm{E}}=\left\{f: f \text { is an } \alpha(/ S) \text {-recursive function from some } \mathbb{N}^{k} \text { into } \mathbb{N}\right\} .
$$

The upper label E refers to Ershov, due to the analogy with the classes of Ershov's hierarchy consisting of just those subsets of $\mathbb{N}^{k}$, which belong to $\Delta_{2}^{0}, c f$. [3,4]. In this sense, we shall also refer to the hierarchy of function classes $\nabla_{\alpha(/ S)}^{\mathrm{E}}$ as the Ershov hierarchy for functions. More precisely, in the sense of Section 1, we would have to write $\nabla_{\alpha(/ S)}^{\mathrm{E}}\left(\mathfrak{F}_{\text {p.r. }}\right)$, where $\mathfrak{F}_{\text {p.r. }}$ denotes the class of all partial recursive functions. Since this basic class is now fixed, however, it is omitted in the denotation.

Obviously, $\nabla_{\alpha(/ S)}^{\mathrm{E}} \subseteq \nabla_{(\alpha+1)(/ S)}^{\mathrm{E}}$ if $S$ is a naming system for $\alpha+1$ too. Let

$$
\nabla_{<\alpha(/ S)}^{\mathrm{E}}=\bigcup_{\beta<\alpha} \nabla_{\beta(/ S)}^{\mathrm{E}} .
$$

For successor numbers $\alpha=\alpha^{\prime}+1$, we have $\nabla_{<\alpha(/ S)}^{\mathrm{E}}=\nabla_{\alpha^{\prime}(/ S)}^{\mathrm{E}}$. For limit numbers $\alpha=\lambda$, the class $\nabla_{<\lambda(/ S)}^{\mathrm{E}}$ is more interesting. The following result shows that strict hierarchy properties are valid w.r.t. the notation-dependent classes.

Proposition 2.1. For all r.r.u. naming systems $(\alpha+1) / S$ and $\lambda / S$, with limit numbers $\lambda$,

$$
\nabla_{\alpha / S}^{\mathrm{E}} \subset \nabla_{(\alpha+1) / S}^{\mathrm{E}} \quad \text { and } \quad \nabla_{<\lambda / S}^{\mathrm{E}} \subset \nabla_{\lambda / S}^{\mathrm{E}}
$$


This follows immediately from analogous results for the classes $\Delta_{\alpha / S}^{\mathrm{E}}$ of Ershov's hierarchy, $c f$. $[3,4,9]$. These classes consist of the so-called $\alpha / S$-recursive subsets of $\mathbb{N}^{k}$, which are characterized by the $\alpha / S$-recursivity of their total characteristic functions in the above defined sense. Thus, due to the classical results, there are even total $\{0,1\}$-valued discrete functions in $\nabla_{(\alpha+1) / S}^{\mathrm{E}} \backslash \nabla_{\alpha / S}^{\mathrm{E}}$ and $\nabla_{\lambda / S}^{\mathrm{E}} \backslash \nabla_{<\lambda / S}^{\mathrm{E}}$, respectively.

The following proposition characterizes the class of functions occurring in the Ershov hierarchy. It also shows that the notation-independent hierarchy collapses at level $\omega^{2}$. Recall that $f \leq_{\mathrm{T}} 0^{\prime}$ means that the function $f$ is $0^{\prime}$-computable, i.e., computable by means of the halting problem as oracle. A function $f: \mathbb{N}^{k} \succ \mathbb{N}$ is called limit-computable iff there is a total recursive function $\varphi: \mathbb{N}^{k+1} \longrightarrow \mathbb{N}$ such that $f(\vec{x}) \simeq \lim _{n \rightarrow \infty} \varphi(\vec{x}, n)$; in particular, $f(\vec{x}) \downarrow$ iff the sequence $(\varphi(\vec{x}, n))_{n<\omega}$ converges.

Proposition 2.2. For any function $f: \mathbb{N}^{k} \succ \mathbb{N}$ are equivalent:

(i) $f \in \nabla_{\alpha / S}^{\mathrm{E}}$, for a constructive ordinal $\alpha$ and a r.r.u. system $\alpha / S$;

(ii) $f \leq_{\mathrm{T}} 0^{\prime}$ and $\operatorname{dom}(f)$ is r.e.;

(iii) $f$ is limit-computable and $\operatorname{dom}(f)$ is r.e.;

(iv) $f \in \nabla_{\omega^{2}}^{\mathrm{E}}$.

The equivalence (ii) $\Leftrightarrow$ (iii) is well-known, $c f$. [21]. (iv) $\Rightarrow$ (i) is trivial, and (i) $\Rightarrow$ (iii) is easily shown. Also, (i) $\Rightarrow$ (ii) was shown in [3]. Finally, (iii) $\Rightarrow$ (iv) was proved in [3] too. (In fact, there it was claimed that $f \in \nabla_{\omega^{2}}^{\mathrm{E}}$ for arbitrary limitcomputable partial functions, the mistake, however, is easily seen.)

\section{The HAUsdorfF hIERARCHY FOR REAL FUnCTIONS}

Hausdorff's difference hierarchy within the Borel class $\Delta_{2}^{\mathrm{B}}$ over (complete and separable) topological spaces was essentially established in [5], long before notions of recursivity or computability came into use. This topological setting, however, was mainly directed to classes of pointsets and is not directly transferable to function classes. Moreover, in topology it is less customary to deal with partial functions, a basic feature in applying the $\Phi$ operator. A considerable progress to a related classification of functions was made by Hertling and Weihrauch, and Hertling, who introduced and studied the levels of discontinuity of functions, see [10-12]. Finally, in [9] we have shown how the Hausdorff hierarchy of classes of pointsets can be characterized by means of the first-value operator. This setting is now transferred to a classification of certain real-valued functions over Euclidean spaces.

In the sequel, by a real function, we mean any partial function $f: \mathbb{R}^{k} \succ \mathbb{R}$, for an arbitrary dimension $k \in \mathbb{N}_{+}$. A real function $f$ is said to be continuous iff for all open $G \subseteq \mathbb{R}$ the preimages $f^{-1}[G]$ are open pointsets too. This means that $f$ has to be continuous in all points $\vec{x} \in \operatorname{dom}(f)$ and, moreover, $\operatorname{dom}(f)=f^{-1}[\mathbb{R}]$ has to be open. The basic class of the hierarchy we are going to introduce will be 
that of continuous functions,

$$
\mathfrak{F}_{\text {cont }}=\{f: f \text { is a continuous real function }\} .
$$

For an ordinal $\alpha \in \mathrm{C}_{\mathrm{II}}$, a real function $f$ is said to be $\alpha$-continuous iff there is an $\alpha$-sequence $\mathcal{F}=\left(f_{\xi}\right)_{\xi<\alpha}$ of continuous functions $f_{\xi} \in \mathfrak{F}_{\text {cont }}$ such that $f=\Phi(\mathcal{F})$. Notice that then $\operatorname{dom}(f)=\bigcup_{\xi<\alpha} \operatorname{dom}\left(f_{\xi}\right)$ is open too. The 1-continuous functions are just the members of $\mathfrak{F}_{\text {cont }}$.

The classes of the Hausdorff hierarchy for real functions are defined as

$$
\begin{gathered}
\nabla_{\alpha}^{\mathrm{H}}=\{f: f \text { is an } \alpha \text {-continuous real function }\} \text { and } \\
\nabla_{<\alpha}^{\mathrm{H}}=\bigcup_{\beta<\alpha} \nabla_{\beta}^{\mathrm{H}} .
\end{gathered}
$$

We have the following strict inclusions.

Proposition 3.1. For all ordinals $\alpha$ and limit numbers $\lambda$ with $\alpha, \lambda \in \mathrm{C}_{\mathrm{II}}$,

$$
\nabla_{\alpha}^{\mathrm{H}} \subset \nabla_{(\alpha+1)}^{\mathrm{H}} \quad \text { and } \quad \nabla_{<\lambda}^{\mathrm{H}} \subset \nabla_{\lambda}^{\mathrm{H}} .
$$

This follows from related properties of the usual Hausdorff hierarchy for the classes $\Delta_{\alpha}^{\mathrm{H}}$, cf. [9], Sections 3 and 5. These classes consist of the $\alpha$-clopen pointsets $A$, which are just those whose total characteristic functions $\chi_{A}$ are $\alpha$-continuous. Thus, there are total $\{0,1\}$-valued real functions in $\nabla_{(\alpha+1)}^{\mathrm{H}} \backslash \nabla_{\alpha}^{\mathrm{H}}$ and $\nabla_{\lambda}^{\mathrm{H}} \backslash \nabla_{<\lambda}^{\mathrm{H}}$, respectively.

Another characterization of the Hausdorff hierarchy of functions can be obtained by the technique of depth analysis. It goes also back to Hausdorff [5], who showed that the resolvable sets are exactly the members of $\Delta_{2}^{\mathrm{B}}$. In $[8,9]$, we have combined his method with features of Ershov's hierarchy [4] within $\Delta_{2}^{0}$. Before that, Hertling and Weihrauch [12], and Hertling [10,11], had applied Hausdorff's method to functions over topological spaces and introduced related levels of discontinuity. In this paper, we essentially follow their setting, even if the notations are modified in order to remain in accordance with [9] and to prepare for a smooth passage to the effective version in the following section, which has not yet been considered so far.

For a closed set $F \subseteq \mathbb{R}^{k}$ and an arbitrary $A \subseteq F$, let $\left.A\right|_{F} ^{\circ}$ denote the interior of $A$ relatively to $F$ which is considered as a complete subspace of $\mathbb{R}^{k}$. Thus, $\left.A\right|_{F} ^{\circ}=\bigcup\left\{B \cap F: B \cap F \subseteq A\right.$, and $B$ is open in $\left.\mathbb{R}^{k}\right\}$.

Given a real function $f: \mathbb{R}^{k} \succ \mathbb{R}$, let the pointsets $C_{f}^{\xi}, U_{f}^{\xi} \subseteq \mathbb{R}^{k}$, for all ordinals $\xi$, be defined by transfinite recursion as follows:

$$
C_{f}^{0}=\left.\{\vec{x}: f(\vec{x}) \downarrow, \text { and } f \text { is continuous in } \vec{x}\}\right|_{\mathbb{R}^{k}} ^{\circ},
$$

this is the ordinary interior of the domain of continuity of function $f$. For $\xi>0$, put $U_{f}^{\xi}=\bigcap_{\eta<\xi} \overline{C_{f}^{\eta}}$ and $C_{f}^{\xi}=\left.\left\{\vec{x} \in U_{f}^{\xi}: f(\vec{x}) \downarrow\right.$, and $\left.f\right|_{U_{f}^{\xi}}$ is continuous in $\left.\vec{x}\right\}\right|_{U_{f}^{\xi}} ^{\circ}$ 
Notice that herein and throughout the paper, the overline denotes the complement of a set.

Of course, since $U_{f}^{0}=\bigcap \emptyset=\mathbb{R}^{k}$, the initial step is included in the general recursion step. By transfinite induction, it follows that any union $\bigcup_{\eta<\xi} C_{f}^{\eta}$ is open in $\mathbb{R}^{k}$ and all $U_{f}^{\xi}$ are closed. Moreover, $C_{f}^{\eta} \cap C_{f}^{\xi}=\emptyset$ if $\eta \neq \xi$.

The sequence of universes $U_{f}^{\xi}$ consists of decreasing closed subsets of $\mathbb{R}^{k}$ all including $\overline{\operatorname{dom}(f)}$,

$$
\mathbb{R}^{k}=U_{f}^{0} \supseteq U_{f}^{1} \supseteq \ldots \supseteq U_{f}^{\xi} \supseteq U_{f}^{\xi+1} \supseteq \cdots \supseteq \overline{\operatorname{dom}(f)} .
$$

Thus, there is a least ordinal $\alpha \in \mathrm{C}_{\mathrm{II}}$ such that $U_{f}^{\alpha}=U_{f}^{\alpha+1}$ or, equivalently, $C_{f}^{\alpha}=\emptyset$. Then we have $U_{f}^{\eta} \supset U_{f}^{\xi}$ and $C_{f}^{\eta} \neq \emptyset$ for all $\eta<\xi \leq \alpha$, but $U_{f}^{\alpha}=U_{f}^{\xi}$ and $C_{f}^{\xi}=\emptyset$ for all $\xi \geq \alpha$.

The function $f$ is called resolvable iff $U_{f}^{\alpha}=\overline{\operatorname{dom}(f)}$ for this least ordinal $\alpha$, at which the sequence of universes becomes stationary. In this case, we have

$$
\operatorname{dom}(f)=\bigcup_{\xi<\alpha} C_{f}^{\xi}
$$

and we define the local depth of a point $\vec{x} \in \operatorname{dom}(f)$ w.r.t. function $f$ by

$$
\operatorname{depth}_{f}(\vec{x})=\xi \text { iff } \vec{x} \in C_{f}^{\xi},
$$

whereas the global depth of $f$ is given by

$$
\operatorname{udepth}(f)=\min \left\{\xi: U_{f}^{\xi}=\overline{\operatorname{dom}(f)}\right\} .
$$

Now we are going to explore the relationships between resolvability and $\alpha$ continuity.

Lemma 3.2. Let $f=\Phi(\mathcal{F})$ with $\mathcal{F}=\left(f_{\xi}\right)_{\xi<\alpha}, f_{\xi} \in \mathfrak{F}_{\text {cont }}, \alpha \in \mathrm{C}_{\mathrm{II}}$. Then, for all ordinals $\xi<\alpha$,

$$
\bigcup_{\eta \leq \xi} \operatorname{dom}\left(f_{\eta}\right) \subseteq \bigcup_{\eta \leq \xi} C_{f}^{\eta}
$$

The inclusion holds for $\xi=0$, i.e., $\operatorname{dom}\left(f_{0}\right) \subseteq C_{f}^{0}$, since $C_{f}^{0}$ is the largest open set on which $f$ is continuous, $f_{0}$ coincides with $f$ on $\operatorname{dom}\left(f_{0}\right)$ and $f_{0}$ is continuous.

Now let the assertion be fulfilled for all $\xi^{\prime}<\xi$. Then,

$$
U_{f}^{\xi}=\bigcap_{\eta<\xi} \overline{C_{f}^{\eta}}=\overline{\bigcup_{\eta<\xi} C_{f}^{\eta}} \subseteq \overline{\bigcup_{\eta<\xi} \operatorname{dom}\left(f_{\eta}\right)}=\bigcap_{\eta<\xi} \overline{\operatorname{dom}\left(f_{\eta}\right)}
$$

$C_{f}^{\xi}$ is the largest relatively open subset of $U_{f}^{\xi}$ on which $\left.f\right|_{U_{f}^{\xi}}$ is continuous. Moreover, $f_{\xi}(\vec{x})=f(\vec{x})$ for all $\vec{x} \in \operatorname{dom}\left(f_{\xi}\right) \backslash\left(\bigcap_{\eta<\xi} \operatorname{dom}\left(f_{\eta}\right)\right)=\operatorname{dom}\left(f_{\xi}\right) \cap$ $\overline{\bigcap_{\eta<\xi} \operatorname{dom}\left(f_{\eta}\right)} \supseteq \operatorname{dom}\left(f_{\xi}\right) \cap U_{f}^{\xi}$, and $f_{\xi}$ is continuous. Thus, $\operatorname{dom}\left(f_{\xi}\right) \cap U_{f}^{\xi} \subseteq C_{f}^{\xi}$, 
and $\bigcup_{\eta \leq \xi} \operatorname{dom}\left(f_{\eta}\right)=\operatorname{dom}\left(f_{\xi}\right) \cup \bigcup_{\eta<\xi} \operatorname{dom}\left(f_{\eta}\right) \subseteq \operatorname{dom}\left(f_{\xi}\right) \cup \bigcup_{\eta<\xi} C_{f}^{\eta} \subseteq\left(\operatorname{dom}\left(f_{\xi}\right) \cap\right.$ $\left.U_{f}^{\xi}\right) \cup \bigcup_{\eta<\xi} C_{f}^{\eta} \subseteq \bigcup_{\eta \leq \xi} C_{f}^{\eta}$.

From Lemma 3.2 one obtains the resolvability of any real function, which is $\alpha$-continuous for some $\alpha \in \mathrm{C}_{\mathrm{II}}$. Indeed, if $f=\Phi(\mathcal{F})$ with a sequence $\mathcal{F}=\left(f_{\xi}\right)_{\xi<\alpha}$, $f_{\xi} \in \mathfrak{F}_{\text {cont }}$, then $\bigcup_{\eta<\alpha} \operatorname{dom}\left(f_{\eta}\right)=\operatorname{dom}(f)$. Since $\bigcup_{\eta<\xi} C_{f}^{\eta} \subseteq \operatorname{dom}(f)$ for all ordinals $\xi$, it follows $C_{f}^{\alpha}=\emptyset$. Thus, $f$ is resolvable. Moreover, by Lemma 3.2, udepth $(f)$ is a lower bound of the lengths of sequences of continuous functions, $\mathcal{F}$, with $\Phi(\mathcal{F})=f$.

Now we look for the inverse direction.

Lemma 3.3. If a real function $f$ is resolvable, then there is a sequence $\mathcal{F}=$ $\left(f_{\xi}\right)_{\xi<\operatorname{udepth}(f)}$ such that $f=\Phi(\mathcal{F})$ and, moreover,

$(+) \quad f_{\xi} \in \mathfrak{F}_{\text {cont }}$ and $\bigcup_{\eta \leq \xi} \operatorname{dom}\left(f_{\eta}\right)=\bigcup_{\eta \leq \xi} C_{f}^{\eta}$ for all $\xi<\operatorname{udepth}(f)$.

Put $f_{0}=\left.f\right|_{C_{f}^{0}}$, and the requirement $(+)$ is fulfilled for $\xi=0$.

Suppose $f_{\eta}$ has been defined for all $\eta<\xi$ such that $(+)$ holds. Then we have $\bigcup_{\eta<\xi} \operatorname{dom}\left(f_{\eta}\right)=\bigcup_{\eta<\xi} C_{f}^{\eta}$. By definition of $C_{f}^{\xi}$, there is an open set $G \subseteq \mathbb{R}^{k}$ such that $C_{f}^{\xi}=U_{f}^{\xi} \cap G$, i.e., $G \subseteq \bigcup_{\eta \leq \xi} C_{f}^{\eta} \subseteq \operatorname{dom}(f)$. Moreover, $\left.f\right|_{C_{f}^{\xi}}$ is a continuous function on $C_{f}^{\xi}$, considered as a closed subset of the normal space $G$. Thus, by the Tietze-Urysohn theorem, see e.g. [2], $\left.f\right|_{C_{f}^{\xi}}$ can be extended to a continuous function $f_{\xi}$ over $G$. So we have $\operatorname{dom}\left(f_{\xi}\right)=G$ and $f_{\xi}(\vec{x})=f(\vec{x})$ for all $\vec{x} \in C_{f}^{\xi}$. It follows $\bigcup_{\eta \leq \xi} \operatorname{dom}\left(f_{\eta}\right)=\bigcup_{\eta \leq \xi} C_{f}^{\eta}$, and $(+)$ is fulfilled.

Proposition 3.4. A real function is resolvable iff it is $\alpha$-continuous for some $\alpha \in \mathrm{C}_{\mathrm{II}}$. More precisely, for any $\alpha \in \mathrm{C}_{\mathrm{II}}$,

$$
\nabla_{\alpha}^{\mathrm{H}}=\{f: f \text { is a resolvable real function, and udepth }(f) \leq \alpha\} .
$$

This only summarizes some results obtained so far.

A sequence of real functions, $\mathcal{F}=\left(f_{\xi}\right)_{\xi<\alpha}$, is called greedy iff, for the function $f=\Phi(\mathcal{F})$ and any $\vec{x} \in \operatorname{dom}(f), \vec{x} \in \operatorname{dom}\left(f_{\xi}\right)$ whenever $\operatorname{depth}_{f}(\vec{x})=\xi$.

To give an intuitive background, let us imagine the sequence $\mathcal{F}$ as the description of a determination process in stages for the function $f$, where the stage $\xi$ of determination consists in applying function $f_{\xi}$. According to the first-value operator, $f(\vec{x})$ is just equal to $f_{\xi_{\vec{x}}}(\vec{x})$ if $\xi_{\vec{x}}=\min \left\{\xi: f_{\xi}(\vec{x}) \downarrow\right\}$. Then, due to Lemma 3.2 , by a greedy sequence for $f$, each value $f(\vec{x})$ is just determined at the earliest possible stage.

Notice that the length $\alpha$ of a greedy sequence for function $f$ can obviously be restricted to udepth $(f)$. Now Lemma 3.3 can be expressed as follows.

Corollary 3.5. Any resolvable real function can be obtained as $\Phi(\mathcal{F})$, with a greedy sequence $\mathcal{F}$ of continuous functions.

Finally, we mention a result shown by Hertling [10] for total functions over metric spaces. It stresses that the resolvable functions belong to a rather low level 
of complexity, within the framework of descriptive set theory. To this purpose, the notion of $\Gamma$-measurable function is employed, $c f$. $[13,17]$ : given a class of pointsets, $\Gamma$, a real function is called $\Gamma$-measurable iff $f^{-1}[G] \in \Gamma$ for all open $G \subseteq \mathbb{R}$.

Lemma 3.6. Any resolvable real function is $\Delta_{2}^{\mathrm{B}}$-measurable.

This can be proved by means of set resolvability, $c f$. [10]. Here we give a direct proof by means of Proposition 2.2, which can be effectivized in a rather straightforward way, $c f$. Section 4.

Let $f=\Phi(\mathcal{F})$ with a sequence $\mathcal{F}=\left(f_{\xi}\right)_{\xi<\alpha}$, where $\alpha \in \mathrm{C}_{\mathrm{II}}$ and $f_{\xi} \in \mathfrak{F}_{\text {cont }}$, and let $G \subseteq \mathbb{R}$ be an open set. Then

$$
f^{-1}[G]=\bigcup_{\xi<\alpha}\left(f_{\xi}^{-1}[G] \cap \bigcap_{\eta<\xi} \overline{\operatorname{dom}\left(f_{\eta}\right)}\right) .
$$

The open pointsets $f_{\xi}^{-1}[G]$ are unions of countably many closed sets: $f_{\xi}^{-1}[G]=$ $\bigcup_{i<\omega} F_{\xi, i}$, with closed $F_{\xi, i}$. The sets $F_{\xi}^{\prime}=\bigcap_{\eta<\xi} \overline{\operatorname{dom}\left(f_{\eta}\right)}$ are closed too. Hence

$$
f^{-1}[G]=\bigcup_{\xi<\alpha}\left(\left(\bigcup_{i<\omega} F_{\xi, i}\right) \cap F_{\xi}^{\prime}\right)=\bigcup_{\xi<\alpha} \bigcup_{i<\omega}\left(F_{\xi, i} \cap F_{\xi}^{\prime}\right) .
$$

This means that $f^{-1}[G] \in \Sigma_{2}^{\mathrm{B}}$, as a countable union of closed sets.

A related representation of the complement is obtained analogously. Firstly,

$$
\overline{f^{-1}[G]}=\overline{\operatorname{dom}(f)} \cup\{\vec{x}: f(\vec{x}) \downarrow, f(\vec{x}) \in \bar{G}\} .
$$

The set $\overline{\operatorname{dom}(f)}$ is closed, and

$$
\{\vec{x}: f(\vec{x}) \in \bar{G}\}=\bigcup_{\xi<\alpha}\left(f_{\xi}^{-1}[\bar{G}] \cap \bigcap_{\eta<\xi} \overline{\operatorname{dom}\left(f_{\eta}\right)}\right) .
$$

We have $f_{\xi}^{-1}[\bar{G}]=\operatorname{dom}\left(f_{\xi}\right) \cap \overline{f_{\xi}^{-1}[G]}$. The open sets $\operatorname{dom}\left(f_{\xi}\right)$ are countable unions of closed sets: $\operatorname{dom}\left(f_{\xi}\right)=\bigcup_{i<\omega} F_{\xi, i}$, with closed $F_{\xi, i}$. Hence

$$
\begin{aligned}
\{\vec{x}: f(\vec{x}) \in \bar{G}\} & =\bigcup_{\xi<\alpha}\left(\left(\bigcup_{i<\omega} F_{\xi, i} \cap \overline{f_{\xi}^{-1}[G]}\right) \cap \bigcap_{\eta<\xi} \overline{\operatorname{dom}\left(f_{\eta}\right)}\right) \\
& =\bigcup_{\xi<\alpha} \bigcup_{i<\omega}\left(F_{\xi, i} \cap \overline{f_{\xi}^{-1}[G]} \cap \bigcap_{\eta<\xi} \overline{\operatorname{dom}\left(f_{\eta}\right)}\right) .
\end{aligned}
$$

Thus, we have a representation of $\overline{f^{-1}[G]}$ as a countable union of closed sets, hence it also belongs to $\Sigma_{2}^{\mathrm{B}}$, and we have shown that $f^{-1}[G] \in \Delta_{2}^{\mathrm{B}}$.

For any set $A \in \Delta_{2}^{\mathrm{B}}, A \subseteq \mathbb{R}^{k}$, the real function $f_{A}=A \times\{1\}$ is $\Delta_{2}^{\mathrm{B}}$-measurable. However, it cannot be resolvable if $\operatorname{dom}\left(f_{A}\right)=A \notin \Sigma_{1}^{\mathrm{B}}$. It is an open question whether the conversion of Lemma 3.6 holds for all functions with open domains.

To show this, it would be sufficient to give a proof for total functions. Indeed, supposed that all total $\Delta_{2}^{\mathrm{B}}$-measurable functions are resolvable, the resolvability of all $\Delta_{2}^{\mathrm{B}}$-measurable functions $f$ with open domains follows. This can be shown by means of the shrink function $g_{\mathrm{sh}}: \mathbb{R} \longrightarrow \mathbb{R}$ defined by $g_{\mathrm{sh}}(x)=\frac{x}{1+|x|}$. 
It is a monotonously increasing homeomorphism mapping the whole real line onto the open interval $(-1,1)$ and transforming rational open intervals into rational open intervals, in an easily computable way. So, the composition $g_{\mathrm{sh}} \circ f$ is $\Delta_{2}^{\mathrm{B}}$ measurable too and has the same domain as function $f$. Let

$$
\bar{f}(\vec{x})=\left\{\begin{array}{cl}
g_{\mathrm{sh}} \circ f(\vec{x}) & \text { if } \vec{x} \in \operatorname{dom}(f), \\
2 & \text { if } \vec{x} \notin \operatorname{dom}(f) .
\end{array}\right.
$$

$\bar{f}$ is a $\Delta_{2}^{\mathrm{B}}$-measurable total real function. Thus, by supposition, it is resolvable. Then $\left.\bar{f}\right|_{\operatorname{dom}(f)}$, as a restriction of a resolvable total function to an open set, is resolvable too, and $f=\left.g_{\mathrm{sh}}^{-1} \circ \bar{f}\right|_{\operatorname{dom}(f)}$ is also resolvable.

\section{The HausdorfF-Ershov hierarchy FOR Generalized EFFECTIVE REAL FUNCTIONS}

As in the case of pointsets, the Hausdorff-Ershov hierarchy for functions is obtained by a suitable combination of ingredients both of Ershov's hierarchy for discrete functions and of the Hausdorff hierarchy for real functions. One feature of effectivity, that we take over from Ershov's setting, consists in the restriction to constructive ordinals as order types and indices of sequences of real functions. Moreover, these functions have to be computable now, and this is defined within the framework of computable analysis, cf. [22]. Since several systems of notations are here still in use, and in order to keep this paper self-contained to some extent, we briefly recall some basic ideas and notations. For more details within our framework, see [7-9].

Real numbers (and tuples of them) are represented by fast converging Cauchy sequences of (tuples of) rational numbers. Let be fixed a total standard numbering $\nu_{\mathbb{Q}}=\left(q_{n}: n \in \mathbb{N}\right)$ of the set of rational numbers $\mathbb{Q}=\left\{q_{n}: n \in \mathbb{N}\right\}$. It is extended to $\mathbb{Q}^{k}$ via Cantor's $k$-tuple function such that $\mathbb{Q}^{k}=\left\{\vec{q}_{n}: n \in \mathbb{N}\right\}$. For points of Euclidean spaces, $\vec{x}=\left(x_{1}, \ldots, x_{k}\right) \in \mathbb{R}^{k}$, we prefer the maximum norm $\|\vec{x}\|=\max _{\varkappa=1}^{k}\left|x_{\varkappa}\right|$ instead of the topologically equivalent Euclidean norm. So we have $\|\vec{x}\| \in \mathbb{Q}$ for all $\vec{x} \in \mathbb{Q}^{k}$. The natural topology is generated by the base of all rational open balls, $\operatorname{Ball}_{q}(\vec{x})=\left\{\vec{y} \in \mathbb{R}^{k}:\|\vec{x}-\vec{y}\|<q\right\}, q \in \mathbb{Q}, \vec{x} \in \mathbb{Q}^{k}$. Let a numbering of them, (ball $n: n \in \mathbb{N}$ ), be fixed by ball $\langle n, m\rangle=$ Ball $_{q_{n}}\left(\vec{q}_{m}\right)$, for any dimension $k$ (which is determined by the context), where $\langle\cdot, \cdot\rangle$ denotes Cantor's pairing function.

Computability for partial real functions $f: \mathbb{R}^{k} \longleftrightarrow \mathbb{R}$ can be defined by means of function-oracle Turing machines. This approach is due to Ko and Friedman [15], and Kreitz and Weihrauch [16]. The points $\vec{x} \in \mathbb{R}^{k}$ are represented by (sequences of indices of) effectively converging Cauchy sequences, i.e., by sequences of the set $\mathrm{CF}_{\vec{x}}=\left\{\sigma \in \mathbb{N}^{\omega}:\left\|\vec{q}_{\sigma(n)}-\vec{x}\right\|<2^{-n}\right.$ for all $\left.n \in \mathbb{N}\right\}$. A function-oracle Turing machine (OTM) $\mathcal{M}$ gets a natural number $n$ as input and a sequence $\sigma=\left(i_{0}, i_{1}, i_{2}, \ldots\right) \in \mathbb{N}^{\omega}$ as oracle, and it has produced an output $\mathcal{M}^{\sigma}(n) \in \mathbb{N}$ 
when it halts. In the course of its work, it can put oracle queries " $m$ ?", for $m \in \mathbb{N}$, which are answered by the $(m+1)$ st element $i_{m}$ of the sequence $\sigma$.

A real function $f: \mathbb{R}^{k} \succ \mathbb{R}$ is said to be (approximately) computable iff there is an OTM $\mathcal{M}$ such that for all $\vec{x} \in \mathbb{R}^{k}$ :

(1) if $f(\vec{x}) \downarrow$, then $\mathcal{M}^{\sigma}(n)$ exists for all $\sigma \in \mathrm{CF}_{\vec{x}}$ and $n \in \mathbb{N}$, and $\left(\mathcal{M}^{\sigma}(n)\right)_{n \in \mathbb{N}} \in$ $\mathrm{CF}_{f(\vec{x})}$;

(2) if $f(\vec{x}) \uparrow$ and $\sigma \in \mathrm{CF}_{\vec{x}}$, there is an input $n \in \mathbb{N}$ for which $\mathcal{M}^{\sigma}(n)$ remains undefined.

Ko and Friedman used a more restrictive notion. Instead of condition (2), they required

(2') if $f(\vec{x}) \uparrow$, then $\mathcal{M}^{\sigma}(n)$ remains undefined for all $\sigma \in \mathrm{CF}_{\vec{x}}$ and all $n \in \mathbb{N}$.

We call a function $f$ KF-computable iff there is an OTM $\mathcal{M}$ satisfying conditions (1) and (2').

Recall that $\Pi_{m}^{\mathrm{ta}}$ is the class of complements of members of $\Sigma_{m}^{\mathrm{ta}}, m \in \mathbb{N}_{+}$, and a pointset $A$ belongs to $\Sigma_{m}^{\text {ta }}$ iff there is a total recursive function $\varphi: \mathbb{N}^{m} \longrightarrow \mathbb{N}$ such that

$(*) \quad A= \begin{cases}\bigcup_{n_{1} \in \mathbb{N}} \bigcap_{n_{2} \in \mathbb{N}} \cdots \bigcup_{n_{m} \in \mathbb{N}} \text { ball }_{\varphi\left(n_{1}, n_{2}, \cdots, n_{m}\right)} & \text { if } m \text { is odd, } \\ \bigcup_{n_{1} \in \mathbb{N}} \bigcap_{n_{2} \in \mathbb{N}} \cdots \bigcap_{n_{m} \in \mathbb{N}} \overline{\text { ball }_{\varphi\left(n_{1}, n_{2}, \cdots, n_{m}\right)}} & \text { if } m \text { is even. }\end{cases}$

If $\varphi$ is allowed to be an arbitrary discrete function herein, we have just a typical representation of a Borel set $A \in \Sigma_{m}^{\mathrm{B}}$. Whereas the domains of approximately computable functions form exactly the class $\Pi_{2}^{\mathrm{ta}}$ in the effective Borel hierarchy $\left(\Sigma_{m}^{\mathrm{ta}}: m \in \mathbb{N}_{+}\right)$, the domains of KF-computable functions are just the r.e. open sets, i.e., the members of $\Sigma_{1}^{\mathrm{ta}}$. More precisely, we have

Lemma 4.1. A pointset $A$ is r.e. open iff $A=\operatorname{dom}(f)$ for a KF-computable real function $f$. An approximately computable real function $f$ is KF-computable iff $\operatorname{dom}(f)$ is r.e. open.

The first part is due to Ko and Friedman [15], the second one follows easily.

Due to Lemma 4.1 and since computable functions are continuous on their domains, the KF-computable real functions form an appropriate effective counterpart of the class of continuous functions, $\mathfrak{F}_{\text {cont }}$. Thus, we take

$$
\mathfrak{F}_{\mathrm{KF}}=\{f: f \text { is a KF-computable real function }\}
$$

as the basic class of the effective first-value hierarchy. Effectivity of transfinite sequences of functions from $\mathfrak{F}_{\mathrm{KF}}$ is defined w.r.t. an r.r.u. naming systems $\alpha / S$. More precisely, a sequence of real functions, $\mathcal{F}=\left(f_{\xi}\right)_{\xi<\alpha}$, is said to be $S-K F$ computable iff there is an OTM $\mathcal{M}$ such that, for all points $\vec{x}$ and ordinals $\xi<\alpha$,

(1) if $f_{\xi}(\vec{x}) \downarrow$, then $\mathcal{M}^{\sigma}(\langle m, n\rangle) \downarrow$ for all $\sigma \in \mathrm{CF}_{\vec{x}}$ and all $n \in \mathbb{N}$, where $m=\nu_{S}^{-1}(\xi)$, and it holds $\left(\mathcal{M}^{\sigma}(\langle m, n\rangle)\right)_{n \in \mathbb{N}} \in \mathrm{CF}_{f_{\xi}(\vec{x})} ;$

(2) if $f_{\xi}(\vec{x}) \uparrow$, then $\mathcal{M}^{\sigma}(\langle m, n\rangle) \uparrow$ for all $\sigma \in \mathrm{CF}_{\vec{x}}$, all $n \in \mathbb{N}$ and $m=\nu_{S}^{-1}(\xi)$. Obviously, it follows $f_{\xi} \in \mathfrak{F}_{\mathrm{KF}}$ for all $\xi<\alpha$. 
A real function $f$ is called $\alpha / S$-toprecursive iff $f=\Phi(\mathcal{F})$ for an $S$-KF-computable sequence $\mathcal{F}=\left(f_{\xi}\right)_{\xi<\alpha}$. The notation-independent version, $\alpha$-toprecursivity means $\alpha / S$-toprecursivity w.r.t. some r.r.u. naming system $\alpha / S$. The denotation "toprecursive" is simply a short form of "topologically recursive" indicating computability in the sense of effective analysis w.r.t. the natural topology of Euclidean spaces. The 1-toprecursive functions are just the KF-computable ones. Each $\alpha$ toprecursive function is $\alpha$-continuous. Analogously to the discrete case, it follows that $\operatorname{dom}(f)$ is r.e. open, for any $\alpha$-toprecursive real function $f$.

For constructive ordinals $\alpha$ (and r.r.u. systems $\alpha / S$ ), the notation-dependent and notation-independent, respectively, versions of the Hausdorff-Ershov classes of functions are defined as

$$
\nabla_{\alpha(/ S)}^{\mathrm{HE}}=\{f: f \text { is an } \alpha(/ S) \text {-toprecursive real function }\} .
$$

By reasons of cardinality, we have immediately the strict inclusions $\nabla_{\alpha(/ S)}^{\mathrm{HE}} \subset \nabla_{\alpha}^{\mathrm{H}}$, for $\alpha \neq 0$, and even $\bigcup\left\{\nabla_{\alpha}^{\mathrm{HE}}: \alpha\right.$ is a constructive ordinal number $\} \subset \bigcup_{\alpha \in \mathrm{C}_{\text {II }}} \nabla_{\alpha}^{\mathrm{H}}$. As for the preceding hierarchies of function classes, the results obtained for the related hierarchy of classes of pointsets can immediately be applied to show the strict hierarchy property.

Proposition 4.2. For all constructive ordinals $\alpha$, constructive limits $\lambda$ (and corresponding r.r.u. naming systems $(\alpha+1) / S$ and $\lambda / S$, respectively),

$$
\nabla_{\alpha(/ S)}^{\mathrm{HE}} \subset \nabla_{(\alpha+1)(/ S)}^{\mathrm{HE}} \quad \text { and } \quad \nabla_{<\lambda(/ S)}^{\mathrm{HE}} \subset \nabla_{\lambda(/ S)}^{\mathrm{HE}}
$$

Indeed, a pointset $A \subseteq \mathbb{R}^{k}$ belongs to $\Delta_{\alpha(/ S)}^{\mathrm{HE}}$ in the sense of [9] iff its total characteristic function $\chi_{A}$ belongs to $\nabla_{\alpha(/ S)}^{\mathrm{HE}}$. Thus, by Corollary 2 and Theorem 1 from [9], we get the strict inclusions.

To localize the functions occurring in classes of the Hausdorff-Ershov hierarchy, Lemma 3.6 can straightforwardly be effectivized within the framework of computable analysis. We sketch this briefly. For details of the definitions and proof techniques, the reader is referred to [1].

Let $\mathcal{N}_{m}$ denote the set of all discrete total functions of arity $m, \varphi: \mathbb{N}^{m} \longrightarrow \mathbb{N}$, $m \in \mathbb{N}_{+}$. Notice that the functions $\varphi \in \mathcal{N}_{m}$ can be represented by sequences $\sigma_{\varphi} \in \mathbb{N}^{\omega}$ in a canonical, bijective way. For example, put $\sigma_{\varphi}(n)=\varphi\left(\gamma_{m}(n)\right)$, where $\gamma_{m}$ is an effective standard bijection of $\mathbb{N}$ onto $\mathbb{N}^{m}$. Thus, the sets $A \in \Sigma_{m}^{\mathrm{B}}$ can be represented by the sequences $\sigma \in \mathbb{N}^{\omega}$ too, namely if $\sigma=\sigma_{\varphi}$ for a function $\varphi \in \mathcal{N}_{m}$ satisfying the typical equation $(*)$ given above. Accordingly, a real function $f$ is called effectively $\Sigma_{m}^{\mathrm{B}}$-measurable iff there is a function $g: \mathbb{N}^{\omega} \longrightarrow \mathbb{N}^{\omega}$ (approximately) computable in the related sense such that, whenever a sequence $\sigma$ represents an open set $A \in \Sigma_{1}^{\mathrm{B}}, A \subseteq \mathbb{R}$, the sequence $g(\sigma)$ represents the preimage $f^{-1}[A]$ as a member of $\Sigma_{m}^{\mathrm{B}}$.

Analogously, we call a real function $f$ effectively $\Delta_{m}^{\mathrm{B}}$-measurable iff there are (approximately) computable functions $g, \bar{g}: \mathbb{N}^{\omega} \longrightarrow \mathbb{N}^{\omega}$ such that, whenever a sequence $\sigma$ represents an open set $A \subseteq \mathbb{R}$, the sequence $g(\sigma)$ represents the preimage 
$f^{-1}[A]$ and $\bar{g}(\sigma)$ represents the complement, $\overline{f^{-1}[A]}$, both as a members of $\Sigma_{m}^{\mathrm{B}}$. By the effective analogue of the proof of Lemma 3.6, with some technical effort using tools prepared in [1], one shows

Lemma 4.3. If a real function is $\alpha / S$-toprecursive w.r.t. some r.r.u. naming system $\alpha / S$, then it is effectively $\Delta_{2}^{\mathrm{B}}$-measurable.

For classes of pointsets, relationships between the Hausdorff-Ershov hierarchy and the Ershov hierarchy have been obtained by considering the discrete parts $A \cap \mathbb{N}^{k}$ of pointsets $A \subseteq \mathbb{R}^{k}$. For functions, the situation is not so simple, since the restrictions of real functions $f: \mathbb{R}^{k} \succ \mathbb{R}$ to $\mathbb{N}^{k}$ are not necessarily discrete, i.e., the ranges are not necessarily subsets of $\mathbb{N}$. Conversely, non-empty discrete functions, $f: \mathbb{N}^{k} \succ \mathbb{N}$, if they are considered as real ones, are not continuous in the sense of Section 3, since the domains are not open. Nevertheless, the Ershov hierarchy of discrete functions can be embedded into the Hausdorff-Ershov hierarchy by means of the operator $\varrho$ assigning to a discrete function $f: \mathbb{N}^{k} \succ \mathbb{N}$ the continuous function $\varrho(f): \mathbb{R}^{k} \succ \mathbb{R}$ defined as $\varrho(f)=\bigcup\left\{\operatorname{Ball}_{\frac{1}{3}}(\vec{x}) \times\{f(\vec{x})\}\right.$ : $\vec{x} \in \operatorname{dom}(f)\}$, i.e.,

$$
\varrho(f)(\vec{y}) \simeq\left\{\begin{array}{cl}
f(\vec{x}) & \text { if } \vec{x} \in \mathbb{N}^{k} \text { and } \vec{y} \in \operatorname{Ball}_{\frac{1}{3}}(\vec{x}), \\
\uparrow & \text { otherwise }
\end{array}\right.
$$

Proposition 4.4. For any function $f: \mathbb{N}^{k} \succ \mathbb{N}$ and any r.r.u. naming system $\alpha / S$,

$$
f \in \nabla_{\alpha / S}^{\mathrm{E}} \quad \text { iff } \varrho(f) \in \nabla_{\alpha / S}^{\mathrm{HE}} .
$$

Indeed, for an $S$-computable sequence $\mathcal{F}=\left(f_{\xi}\right)_{\xi<\alpha}$ of discrete functions $f_{\xi}$, the sequence of real functions $\varrho(\mathcal{F})=\left(\varrho\left(f_{\xi}\right)\right)_{\xi<\alpha}$ is $S$-KF-computable. Moreover, $\Phi(\varrho(\mathcal{F}))=\varrho(\Phi(\mathcal{F}))$. Conversely, if $\varrho(f)=\Phi(\mathcal{F})$ for an $S$-KF-computable sequence $\mathcal{F}=\left(f_{\xi}\right)_{\xi<\alpha}$ of $k$-ary real functions, then the sequence $\mathcal{F}^{\prime}=\left(f_{\xi}^{\prime}\right)_{\xi<\alpha}$, where $f_{\xi}^{\prime}=\left.f_{\xi}\right|_{\mathbb{N}^{k}}$, consists of discrete functions and is $S$-computable in the sense of Section 2. Moreover, $\Phi\left(\mathcal{F}^{\prime}\right)=f$.

So, the Hausdorff-Ershov hierarchy of function classes is at least as rich as the related Ershov hierarchy. Propositions 2.1 and 4.4 show that each class $\nabla_{\alpha / S}^{\mathrm{HE}}$ contains continuous functions which do not belong to any lower class $\nabla_{\alpha^{\prime} / S}^{\mathrm{HE}}$ for $\alpha^{\prime}<\alpha$. In other words, for any r.r.u. naming system $\alpha / S$ there are real functions of Hausdorff level $1, f \in \nabla_{1}^{\mathrm{H}}=\mathfrak{F}_{\text {cont }}$, which have the level $\alpha / S$ in the notationdependent Hausdorff-Ershov hierarchy, i.e., $f \in \nabla_{\alpha / S}^{\mathrm{HE}} \backslash \nabla_{<\alpha / S}^{\mathrm{HE}}$. So the level of toprecursivity of a real function can be arbitrarily higher than its level of continuity. (Here it seems indeed to be more appropriate to speak of the levels or degrees of non-toprecursivity and of dis-continuity, respectively.)

Those functions which admit computable greedy sequences, representing them by means of the first-value operator, are of special interest. For such a function, its level in the Hausdorff hierarchy coincides with that in the Hausdorff-Ershov hierarchy. Moreover, for each point $\vec{x}$ from the domain, the computation of the 
function value in $\vec{x}$ can be performed by computing $f_{\xi}(\vec{x})$, just at the first stage $\xi$ which is possible at all, by topological reasons.

So, a real function $f$ is called weakly ( $\alpha / S$-) computable iff there is an $S$-KFcomputable greedy sequence $\mathcal{F}=\left(f_{\xi}\right)_{\xi<\alpha}$ with $f=\Phi(\mathcal{F})$.

A still sharper condition is obtained by requiring, moreover, that the distance errors for any function $f_{\xi}$ of sequence $\mathcal{F}$ can be made arbitrarily small. This means that if $f_{\xi}(\vec{x}) \downarrow$, then there is a point $\vec{y}$ close to $\vec{x}$ such that $\operatorname{depth}_{f}(\vec{y})=\xi$ and, therefore, $f(\vec{y})=f_{\xi}(\vec{y}) \downarrow$.

More precisely, a real function $f$ is called safely $(\alpha / S$-) computable iff there is an $S$-KF-computable double sequence $\mathcal{D}=\left(f_{n, \xi}\right)_{n<\omega, \xi<\alpha}$ such that the sequences $\mathcal{F}_{n}=\left(f_{n, \xi}\right)_{\xi<\alpha}$ are greedy and $\Phi\left(\mathcal{F}_{n}\right)=f$, for all $n \in \mathbb{N}$, and whenever $f_{n, \xi}(\vec{x}) \downarrow$, then there is a point $\vec{y} \in \operatorname{Ball}_{2^{-n}}(\vec{x})$ with $\operatorname{depth}_{f}(\vec{y})=\xi$. S-KF-computability of $\mathcal{D}$ means that there is an OTM $\mathcal{M}$ such that, for all points $\vec{x}$, numbers $n \in \mathbb{N}$ and ordinals $\xi<\alpha$,

(1) if $f_{n, \xi}(\vec{x}) \downarrow$, then $\mathcal{M}^{\sigma}(\langle n, m, l\rangle) \downarrow$ for all $\sigma \in \mathrm{CF}_{\vec{x}}, n, l \in \mathbb{N}$ and $m=$ $\nu_{S}^{-1}(\xi)$, and it holds $\left(\mathcal{M}^{\sigma}(\langle n, m, l\rangle)\right)_{l \in \mathbb{N}} \in \mathrm{CF}_{f_{n, \xi}(\vec{x})}$;

(2) if $f_{n, \xi}(\vec{x}) \uparrow$, then $\mathcal{M}^{\sigma}(\langle n, m, l\rangle) \uparrow$ for all $\sigma \in \mathrm{CF}_{\vec{x}}$, all $n, l \in \mathbb{N}$ and $m=$ $\nu_{S}^{-1}(\xi)$.

In [9], Section 6 , we considered the analogous notions concerning decidability of pointsets $A$, the weak and approximate decidability, which just mean the weak and safe computability, respectively, of the characteristic function $\chi_{A}$. By the examples constructed there, we have

Lemma 4.5. To any r.r.u. naming system $\alpha / S$, there is a real function $f_{\alpha / S}: \mathbb{R} \longrightarrow\{0,1\}$ which is safely $\alpha / S$-computable and has the global depth $\operatorname{udepth}\left(f_{\alpha / S}\right)=\alpha$.

So, $f_{\alpha / S}$ witnesses that there are safely $\alpha / S$-computable, hence also weakly $\alpha / S$-computable functions that are of level $\alpha$ in the (non-effective) Hausdorff hierarchy. From discussions in $[7,9]$ it follows that there are weakly computable (total) functions which are not safely computable.

By means of the notion of r.e. closed set, the safely computable functions can be characterized among the weakly computable ones. Recall that a pointset $A \subseteq \mathbb{R}^{k}$ is said to be r.e. closed iff $A=\emptyset$ or there is a total recursive function $\varphi: \mathbb{N}^{2} \longrightarrow \mathbb{N}$ such that there is a sequence $\left(\vec{x}_{n}\right)_{n \in \mathbb{N}}$ of points $\vec{x}_{n} \in \mathbb{R}^{k}$ satisfying $\left\|\vec{q}_{\varphi(n, m)}-\vec{x}_{n}\right\|<$ $2^{-m}$ for all $n, m \in \mathbb{N}$, and $A=\operatorname{cl}\left(\left\{\vec{x}_{n}: n \in \mathbb{N}\right\}\right)$, where cl denotes the closure of a set.

If a real function $f$ is safely $\alpha / S$-computable, then the closures of the domains of continuity, i.e., the sets $\operatorname{cl}\left(C_{f}^{\xi}\right), \xi<\alpha$, are uniformly r.e. closed. This means that there is a recursive function $\varphi: \mathbb{N}^{3} \succ \mathbb{N}$ such that the functions $\varphi_{\xi}: \mathbb{N}^{2} \succ \mathbb{N}$, defined by $\varphi_{\xi}(n, m) \simeq \varphi\left(\nu_{S}^{-1}(\xi), n, m\right)$, are total and witness that the sets $\operatorname{cl}\left(C_{f}^{\xi}\right)$ are r.e. closed sets in the above sense. This follows easily from the definition of safe computability. 
Conversely, given a weakly $\alpha / S$-computable function $f$ such that the sets $\operatorname{cl}\left(C_{f}^{\xi}\right)$, $\xi<\alpha$ are uniformly r.e. closed, the safe $\alpha / S$-computability can be shown. So we have

Lemma 4.6. A weakly $\alpha / S$-computable function $f$ is safely $\alpha / S$-computable iff the sets $\operatorname{cl}\left(C_{f}^{\xi}\right), \xi<\alpha$, are uniformly r.e. closed.

\section{Concluding Remarks}

The notions and results presented in this paper provide a unified framework for non-effective and effective hierarchies of classes of real functions which all are $\Delta_{2}^{\mathrm{B}}$ measurable and effectively $\Delta_{2}^{\mathrm{B}}$-measurable, respectively. This establishes a useful tool in classifying certain functions both from the viewpoint of descriptive set theory as well as of computable analysis. Nevertheless, we know only some basic facts and relationships so far, whereas a lot of questions has been left open. As an example, we mention the problem whether there are conversions of Lemmas 3.6 and 4.3 , for functions with (r.e.) open domains. This would nicely characterize the functions of the Hausdorff and Hausdorff-Ershov hierarchy, respectively, within the framework of (effective) descriptive set theory.

Acknowledgement. The author would like to thank an anonymous referee for his helpful comments and suggestions concerning an earlier version of this paper.

\section{REFERENCES}

[1] V. Brattka, Effective Borel measurability and reducibility of functions. Math. Logic Quart. 51 (2005) 19-44.

[2] R. Engelking, General topology. Heldermann Verlag, Berlin (1989).

[3] R.L. Epstein, R. Haas and R.L. Kramer, Hierarchies of sets and degrees below 0', in Logic Year 1979/80, Univ. of Connecticut, edited by M. Lerman, J.H. Schmerl and R.I. Soare. Lect. Notes Math. 859 32-48.

[4] Yu. L. Ershov, A hierarchy of sets. I; II; III. Algebra Logica 7 (1968) 15-47. Algebra Logica 7 (1968) 47-74. Algebra Logica 9 (1970) 34-51 (English translation by Plenum P.C.).

[5] F. Hausdorff, Grundzüge der Mengenlehre. W. de Gruyter \& Co., Berlin and Leipzig (1914); Reprint: Chelsea P.C., New York (1949).

[6] F. Hausdorff, Mengenlehre. W. de Gruyter \& Co., Berlin and Leipzig (1927).

[7] A. Hemmerling, Approximate decidability in Euclidean spaces. Math. Logic Quart. 49 (2003) $34-56$.

[8] A. Hemmerling, Characterizations of the class $\Delta_{2}^{\text {ta }}$ over Euclidean spaces. Math. Logic Quart. 50 (2004) 507-519.

[9] A. Hemmerling, The Hausdorff-Ershov hierarchy in Euclidean spaces. Arch. Math. Logic 45 (2006) 323-350.

[10] P. Hertling, Unstetigkeitsgrade von Funktionen in der effektiven Analysis. Dissertation. Informatik Berichte 208-11/1996, Fern-Uni Hagen (1996).

[11] P. Hertling, Topological complexity with continuous operations. J. Complexity 12 (1996) 315-338. 
[12] P. Hertling and K. Weihrauch, Levels of degeneracy and exact lower complexity bounds for geometric algorithms, in Proc. of the 6th Canadian Conf. on Computational Geometry, Saskatoon (1994) 237-242.

[13] A.S. Kechris, Classical descriptive set theory. Springer Verlag, New York (1995).

[14] K.-I. Ko, Complexity theory of real functions. Birkhäuser, Boston (1991).

[15] K.-I. Ko and H. Friedman, Computational complexity of real functions. Theoret. Comput. Sci. 20 (1982) 323-352.

[16] C. Kreitz and K. Weihrauch, Complexity theory on real numbers and functions. Lect. Notes. Comput. Sci. 145 (1982) 165-174.

[17] Y.N. Moschovakis, Descriptive set theory. North-Holland, Amsterdam (1980).

[18] P. Odifreddi, Classical recursion theory. North-Holland, Amsterdam (1989).

[19] H. Rogers Jr., Theory of recursive functions and effective computability. McGraw-Hill, New York (1967).

[20] V.L. Selivanov, Wadge degrees of $\omega$-languages of deterministic Turing machines. RAIROTheor. Inf. Appl. 37 (2003) 67-84.

[21] R.I. Soare, Recursively enumerable sets and degrees. Springer-Verlag, Berlin (1987).

[22] K. Weihrauch, Computable analysis. Springer-Verlag, Berlin (2000).

Communicated by C. Choffrut.

Received June 21, 2006. Accepted February 1, 2007. 